\title{
Detección y caracterización del circovirus porcino tipo 2 (PCV2) circulante en cerdos de los departamentos de Tolima y Huila, Colombia
}

\section{Detection and characterization of porcine circovirus type 2 (PCV2) circulating in pigs of the departments of Tolima and Huila, Colombia}

\author{
Geiner Almario-Leiva ${ }^{1}$, Rafael Suarez-Mesa ${ }^{1}$, Fabián Uribe-García ${ }^{1}$, \\ Iang Rondón-Barragán ${ }^{1,2,3}$
}

\section{Resumen}

El objetivo del presente trabajo fue evidenciar la presencia del circovirus porcino tipo 2 (PCV2) y la serorreactividad en granjas porcícolas en las regiones de Tolima y Huila, Colombia, mediante un estudio transversal. Se tomaron muestras de sangre y tejidos (linfonodos, pulmón y riñón) de animales de todas las etapas productivas, incluyendo animales sanos y con signos de la enfermedad. Mediante la prueba de ELISA se detectaron animales seropositivos en todas las etapas productivas. Para identificar la presencia del virus se amplificó el ORF2 completo del PCV2 mediante PCR punto final utilizando ADN extraído de sangre, linfonodos, pulmón y riñón. Las muestras fueron genotipificadas, encontrándose animales positivos a PCV2, tanto sanos como enfermos, en todas las etapas de producción. El análisis de las secuencias demostró un porcentaje de identidad entre 93 y $99 \%$ con el genotipo PCV2d. Se encontraron animales sin signos clínicos positivos a la prueba de ELISA y al PCR, lo cual sugiere la presencia de infecciones subclínicas o variaciones en la virulencia de las cepas infectantes. Adicional a estas pruebas, se realizó una encuesta epidemiológica, donde no se detectaron asociaciones significativas entre la presencia del virus y el manejo de la producción.

Palabras clave: diagnóstico, PCV2, enfermedad asociada a circovirus porcino, PCVAD

\footnotetext{
${ }^{1}$ Grupo de Investigación en Inmunobiología y Patogénesis, Facultad de Medicina Veterinaria y Zootecnia, Universidad del Tolima, Ibagué, Tolima, Colombia

${ }^{2}$ Grupo de Investigación en Avicultura, Facultad de Medicina Veterinaria y Zootecnia, Universidad del Tolima, Ibagué, Tolima, Colombia

${ }^{3}$ E-mail: isrondon@ut.edu.co
}

Recibido: 3 de abril de 2019

Aceptado para publicación: 3 de enero de 2020

Publicado: 31 de marzo de 2020 
The aim of this study was to evaluate the presence of the porcine circovirus type 2 (PCV2) and the seroreactivity in pig farms in the regions of Tolima and Huila, Colombia, through a cross-sectional study. Blood and tissue samples (lymph nodes, lung and kidney) were taken from animals of all productive stages, including healthy animals and those with signs of the disease. The ELISA test detected seropositive animals in all productive stages. To identify the presence of the virus, the complete ORF2 of PCV2 was amplified by endpoint PCR using DNA extracted from blood, lymph nodes, lung and kidney. The samples were genotyped and finding positive animals for PCV2, both healthy and diseased, at all stages of production. Sequence analysis demonstrated a percentage of identity between 93 and $99 \%$ with the PCV2d genotype. Animals without positive clinical signs were positive for the ELISA test and for the PCR, which suggests the presence of subclinical infections or variations in the virulence of the infecting strains. In addition to these tests, an epidemiological survey was conducted, where no significant associations were detected between the presence of the virus and production management.

Key words: diagnosis, PCV2, porcine circovirus-associated disease, PCVAD

\section{INTRODUCCIÓN}

La circovirosis porcina es una enfermedad de distribución mundial causada por el circovirus porcino tipo 2 (PCV2), un virus de ADN de la familia Circoviridae (Todd et al., 1991) que afecta tanto a cerdos jóvenes como adultos, produciendo un fuerte impacto económico sobre la producción porcina (Alarcon et al., 2013; Zaveckas et al., 2015). El PCV2 se ha asociado con la mayoría de los casos clínicos de cerdos, especialmente con el síndrome del desmedro multisistémico posdestete (PMWS) (Grau-Roma et al., 2008; Ellis, 2014), el síndrome de la dermatitis y nefropatía porcina (PDNS) y algunos trastornos reproductivos (Olvera et al., 2007; Zhao et al., 2014), los cuales se han nombrado de manera colectiva como enfermedades asociadas al circovirus porcino (PCVAD).

La presencia de PCV2 en células de la médula ósea ha sido relacionada con infección persistente en cerdos que permitiría el mantenimiento y propagación del virus en las granjas porcinas (Wang et al., 2013; Kweon et al., 2015). Recientemente, el PCV3 ha sido descrito en varios países asociado a inflamación multisistémica, fallas reproductivas y PDNS (Zhai et al., 2017; Hayashi et al., 2018; Klaumann et al., 2018; Sukmak et al., 2019) en algunos casos en coinfección con bacterias (Hayashi et al., 2018) y virus, incluyendo el PCV2 (Li et al., 2018).

Diversos trabajos han demostrado una amplia diversidad genética presente en el PCV2, adscrita principalmente a su naturaleza de virus de $\mathrm{ADN}$ de cadena simple con una alta tasa de sustitución de nucleótidos y una alta probabilidad de mutaciones (Firth et al., 2009). PCV2a fue el genotipo más prevalente en cerdos clínicamente afectados, después de lo cual el PCV2b fue el más predominante y nuevos cambios en esa predominancia han sido asociados al uso de vacunas contra PCV2 a nivel mundial (Franzo y Segalés, 2018). No obstante, Franzo et al. (2015) demostraron que ocho cepas obtenidas de cerdos silvestres en Brasil pertenecían a cuatro genotipos: PCV2a, PCV2b, PCV2c y PCV2d. Sumado a esta diversidad, se han descrito que algunas mutaciones del PCV2 han demostrado generar una mayor 
patogenicidad en las cepas virales (Opriessnig et al., 2014). Así, el PCV2 cuenta con genotipos a, b, c, d, e y f (Wei et al., 2013; Neira et al., 2017; Correa-Fiz et al., 2018) y recientemente estudios filogenéticos proponen los genotipos PCV2g y PCV2h, basados en criterios de distancias genéticas entre los clusters (Franzo y Segalés, 2018).

En Colombia, algunos estudios han demostrado la presencia de PCV2 en varias regiones del país (Rincón-Monroy et al., 2015). Sin embargo, se desconoce la presencia del PCV2 en las regiones del Tolima y Huila, las cuales representan parte del suroccidente y centro-oeste del país. El objetivo del presente estudio fue detectar y caracterizar de manera preliminar el circovirus porcino tipo 2 (PCV2) en granjas porcícolas de los departamentos de Huila y Tolima, Colombia.

\section{Materiales y Métodos}

\section{Granjas Porcícolas}

Se llevó a cabo un estudio transversal en cinco granjas porcícolas comerciales del- departamento del Huila, en el suroccidente de Colombia y en otras tres granjas del departamento del Tolima en el centro-oeste del país. En estas granjas se tomaron muestras, sin tener en cuenta la línea genética. Los animales eran alimentados durante todo su ciclo productivo con concentrado comercial.

\section{Animales y Toma de Muestras}

Se tomaron muestras de sangre de 20 cerdas reproductoras de diferentes edades una semana antes del parto, de 20 lechones destetos entre 5 y 10 semanas de vida, de 20 cerdos de levante entre 11 y 16 semanas y de 20 cerdos cebados entre 17 y 23 semanas de cada granja (Figura 1). En ninguna de las granjas se realizaba la vacunación contra el circovirus porcino. Los animales fueron seleccionados aleatoriamente entre cerdos sanos y con signos clínicos relacionados con enfermedades asociadas al circovirus porcino (PCVAD). Las muestras fueron colectadas de la vena yugular mediante sistema BD Vacutainer ${ }^{\circledR}$, siguiendo métodos de recolección estándar, minimizando el daño o estrés a los animales (Uhart et al., 2016) para la obtención de sangre completa y suero.
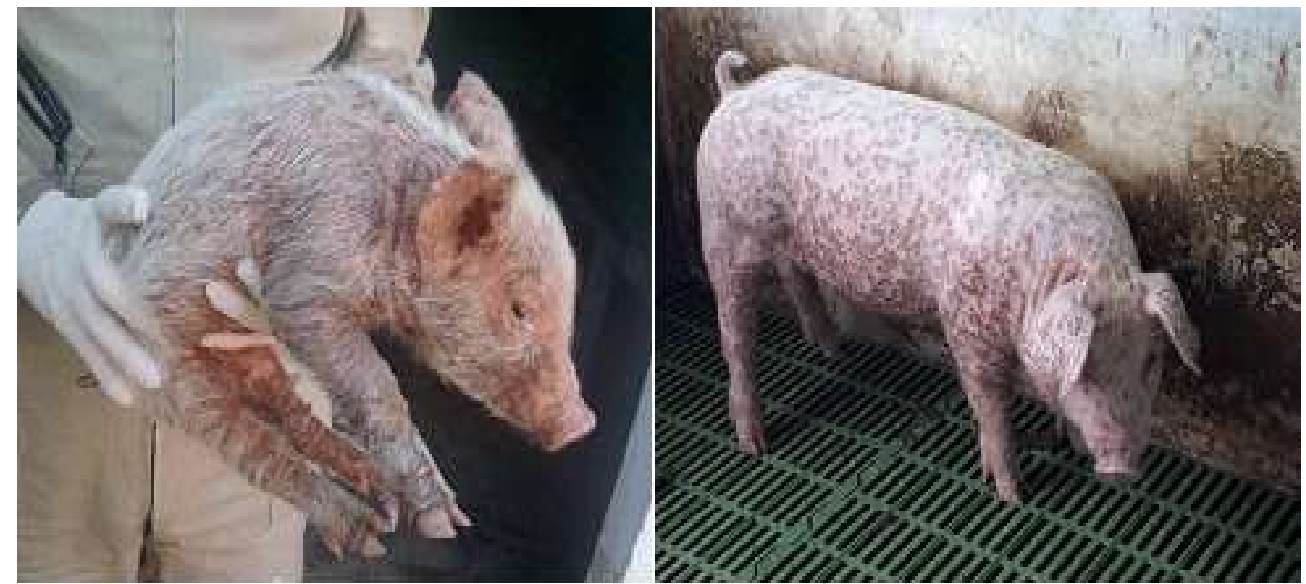

Figura 1. Animales positivos a PCV2 muestreados en granjas del Tolima y Huila. Izquierda: Lechón con signos respiratorios y nerviosos leves, con retraso en el crecimiento comparado con la camada, siendo un animal compatible con PMWS. Presentaba pelo hirsuto y costras en la piel. Derecha: Animal adulto con lesiones cutáneas compatibles con circovirosis porcina 


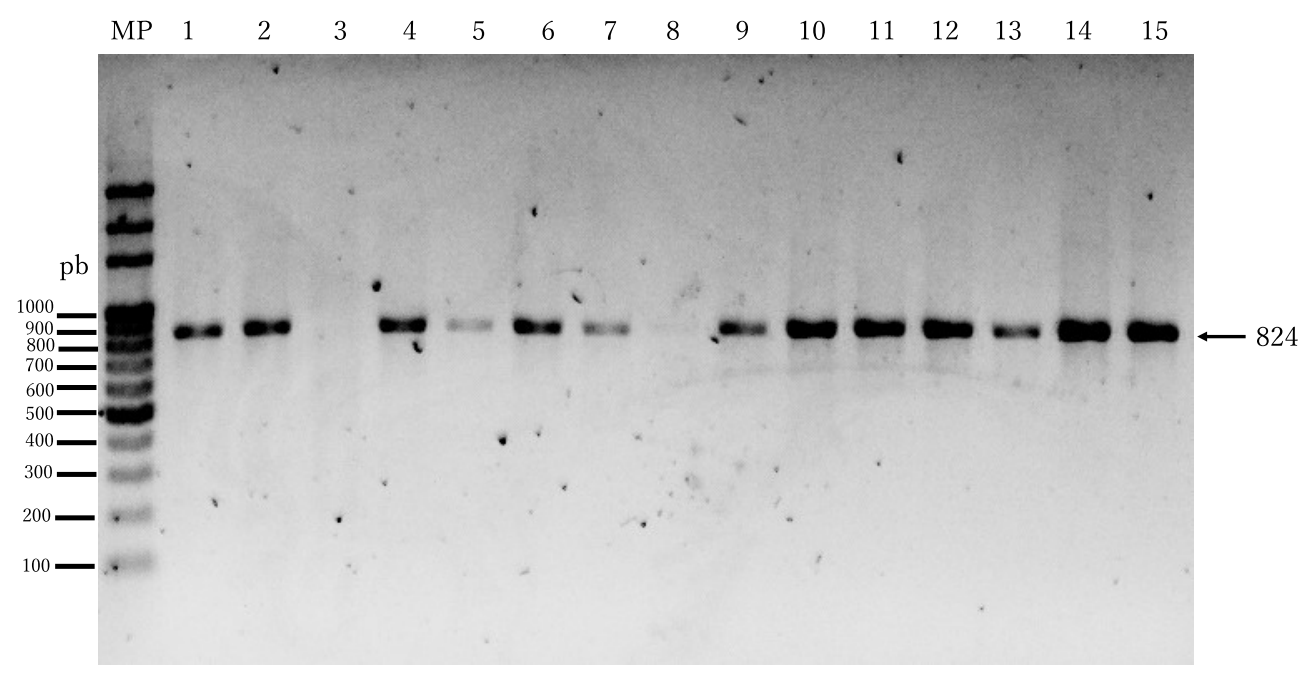

Figura 2. Gel de agarosa mostrando la amplificación del ORF2 del PCV2 mediante PCR convencional de algunas muestras representativas de tejidos de cerdos de los departamentos del Tolima y Huila, Colombia. MP: marcador de peso (100 pb, MiniPCR, Amplyus, USA), 1-15: muestras. La muestra 3 fue negativa, la muestra 8 presenta una banda tenue, confirmada con el secuenciamiento

Se crearon pooles de sueros de cinco animales (Nielsen et al., 2018), los cuales fueron enviados al Laboratorio de Diagnóstico Veterinario del Instituto Colombiano Agropecuario (ICA) para la medición de anticuerpos circulantes por medio de la técnica de ELISA (McNeilly et al., 2002). En los predios que se hallaron animales con signos clínicos compatibles con circovirosis porcina asociados a cuadros respiratorios $y$ cutáneos severos, se realizaron necropsias y se tomaron muestras de órganos (i.e. linfonodos, pulmón, riñón) para la detección del PCV2.

Adicionalmente, se realizó una encuesta epidemiológica de aspectos básicos de manejo de la producción como la bioseguridad, nutrición, reproducción, haciendo especial énfasis en los aspectos sanitarios, con el fin de establecer los posibles factores de riesgo, mediante el análisis de tablas de contingencia y el cálculo del odds ratio. Para ello se empleó el software GraphPad Prism v. 6 para
MacOS (Graph Pad software, San Diego, USA).

\section{Detección Molecular de PCV2 median- te PCR y Secuenciamiento}

Se realizó extracción de ADN total a partir de sangre completa y tejidos de diversos órganos mediante el kit Invisorb ${ }^{\circledR}$ Spin Universal (STRATEC Molecular, Alemania) siguiendo el procedimiento recomendado por el fabricante. El PCV2 fue detectado en los tejidos mediante amplificación de un fragmento de 657 pares de bases (pb) utilizando cebadores (primers) específicos (FGCCAGTTCGTCACCCTTT, R-CTCCCGCACCTTCGGATAT) para el virus (Quintana et al., 2002). Adicionalmente, se amplificó el marco de lectura abierto 2 (ORF2) completo del virus, el cual codifica para la proteína de cápside viral (Nawagitgul et al., 2000), mediante los cebadores F-GCCAGTTCGTCACCCTTT y RCAGC-GCACTTCTTTCGTT, basados en la secuencia de la 
cepa Zhuji2003 (número de acceso AY579893), con un tamaño de amplicón de 824 pb. La reacción de PCR se llevó a cabo en un termociclador T-100 (Bio-Rad, USA) usando un volumen final de reacción de $25 \mu 1$, compuesto por $14.87 \mu 1$ de agua destiladadesionizada, $5 \mu$ de $5 x$ Colorless GoTaq ${ }^{\circledR}$ Flexi Buffer, $1 \mu 1$ de dNTPs $(1.5 \mathrm{mM}), 1 \mu 1$ de cada cebador (forward y reverse) $(10 \mathrm{pmol} / \mu \mathrm{l})$, $1 \mu 1 \mathrm{MgCl}_{2}(25 \mathrm{mM}), 0.125 \mu 1 \mathrm{de} \mathrm{GoTaq}^{\circledR}$ Flexi DNA polymerase (Promega, Madison, USA) y $1 \mu 1$ de la muestra de ADN genómico.

La amplificación consistió en un ciclo de desnaturalización inicial a $95^{\circ} \mathrm{C}$ por $3 \mathrm{~min}$, seguida de 35 ciclos de desnaturalización a $95{ }^{\circ} \mathrm{C}$ por $30 \mathrm{~s}$, anillaje a $55^{\circ} \mathrm{C}$ por $30 \mathrm{~s}$, extensión a $72^{\circ} \mathrm{C}$ por $60 \mathrm{~s}$ y el último paso de extensión final a $72{ }^{\circ} \mathrm{C}$ por $7 \mathrm{~min}$. Los amplicones fueron revelados mediante electroforesis horizontal en gel de agarosa al $1 \%$, teñidos con Hydragreen ${ }^{\circledR}$ (ACTGene, USA) y visualizados bajo la luz ultravioleta mediante un documentador de geles ENDURO GDS ${ }^{\text {TM }}$ (LabNet Intl, USA). Los amplicones del ORF2 completo fueron secuenciados a través del secuenciamiento de Sanger en Macrogen (Seoul, Korea), sometidos a análisis bioinformático, mediante el software Geneious v. 8.1.9 (Biomatters) (Kearse et al., 2012) y comparados con lo reportado en la base de datos del GenBank (National Center for Biotechnology Information - NCBI, USA).

\section{Resultados y Discusión}

El análisis serológico demostró la presencia de animales en diferentes etapas de producción que se encontraban seropositivos al PCV2 $(n=25 ; 15$ etapa de ceba provenientes del Huila, 10 etapa de levante provenientes del Tolima), con títulos de anticuerpos que demuestran una infección anterior o contacto con el virus. Todos los animales seropositivos fueron positivos a PCV2 mediante prueba de PCR. En una de las granjas, los títulos de anticuerpos demostraron la posibilidad de una infección activa. Estos ha- llazgos concuerdan con los reportes de seroprevalencia realizados por Liu et al. (2002) y Mesu et al. (2000) quienes demostraron que animales sanos, sin lesiones discernibles de alguna forma clínica de circovirosis, muestran títulos de anticuerpos considerados como positivos o expuestos. Así mismo, Larochelle et al. (2003) demostraron que los perfiles serológicos de animales sanos fueron iguales a los de aquellos animales con PMWS. Por otro lado, se ha descrito que esta serorreactividad está ampliamente relacionada con la cercanía a zonas o áreas donde el virus es endémico o está circulante y haya una posible transmisión (Mesu et al., 2001; Liu et al., 2002).

En el presente estudio se evaluó la presencia de nucleótidos virales en suero y tejidos de animales sanos y enfermos; no obstante, no fue posible su detección en algunas muestras de suero, por lo que se optó por el uso de sangre completa para la extracción de ADN y amplificación a través de PCR (Figura 2), lo cual arrojó algunos animales positivos en las mismas muestras detectadas como negativas previamente. En el trabajo de Liu et al. (2002) se ha descrito que cerdos infectados experimentalmente muestran niveles de nucleótidos detectables en los tejidos y que pueden no producir una señal detectable en sangre. Adicionalmente, se considera que pese a encontrarse el PCV2 en plasma/suero y a través de las células sanguíneas, su predilección por replicación en macrófagos/ monocitos (Sun et al., 2016) hace más factible utilizar fracciones celulares para la detección de los antígenos virales. En el presente estudio, la presencia de animales positivos a PCV2 sin signos clínicos compatibles con circovirosis porcina sugiere la presencia de infecciones subclínicas o variaciones en la virulencia de las cepas infectantes (Larochelle et al., 2003; Aiki-Raji et al., 2018).

Las muestras que resultaron positivas a la amplificación del ORF2 de PCV2 fueron secuenciadas y comparadas con secuencias de referencia depositadas en el GenBank. 
Todas las muestras enviadas a secuenciar fueron confirmadas como PCV2 ORF2 y correspondieron al PCV2d, mostrando un porcentaje de identidad de nucleótidos entre ellas y las depositadas en las bases de datos de un $93-99 \%$. Estos hallazgos difieren con aquellos de Rincón-Monroy et al. (2014) para los departamentos del Cundinamarca, Valle, Antioquia y Risaralda en Colombia, donde se demostró la presencia de PCV2 de diferentes genotipos, en su mayoría el $2 b$.

En el presente estudio se realizaron encuestas epidemiológicas tendientes a establecer factores de riesgo asociados a la presencia del PCV2; sin embargo, no se detectaron asociaciones significativas entre la positividad al PCV2 y las características de producción y manejo de los cerdos en la granja (datos no mostrados), lo cual puede deberse al bajo número de unidades de producción muestreadas. En Colombia se han establecido las buenas prácticas de producción que deben seguir las ganaderías en general y la producción porcina en particular, siendo claro que el cumplimento del componente de bioseguridad y sanitario reduce el riesgo de transmisión de microorganismos potencialmente patógenos. Este incluye la desinfección, sistemas todo dentro todo fuera, restricción de movimiento de animales entre producciones, entre otros (ICA, 2011). Sin embargo, en países con alto nivel de tecnificación se han demostrado valores altos de prevalencia a PCV2, lo cual ha llevado a pensar que la restricción de movimiento de los animales no está reduciendo la diseminación del PCV2 (Liu et al., 2002) y se deben buscar programas de vigilancia activa que permitan un seguimiento, no solo a los animales a movilizar, sino también a la dinámica de la infección dentro de una área o región.

\section{Conclusiones}

En las granjas porcícolas de las regiones del Tolima y Huila en Colombia se encuentra circulante el circovirus porcino tipo 2d (PCV2d), tanto de manera clínica como subclínica. La presencia del virus en animales clínicamente sanos y en granjas sin vacunación contra el virus denotan la circulación del virus en estas regiones y demanda estudios complementarios de vigilancia activa y caracterización epidemiológica de esta infección.

\section{Agradecimientos}

Los autores agradecen al Sistema General de Regalías por el apoyo al desarrollo del Proyecto «Detección y caracterización del circovirus porcino tipo 2 (PCV2) circulante en cerdos del Departamento del Tolima y Huila», Código 400220516, dentro del Proyecto Macro «Implementación de una estrategia para optimizar las capacidades científicas y tecnológicas del departamento del Tolima» EOCYT. Así mismo, agradecen a la Oficina Central de Investigaciones de la Universidad del Tolima.

\section{Literatura Citada}

1. Aiki-Raji CO, Adebiyi AI, Oluwayelu DO. 2018. A slaughterhouse survey for porcine circovirus type 2 in commercial pigs in Ibadan, Southwest Nigeria. Folia Vet 62:30-34. doi: 10.2478/fv-2018-0014

2. Alarcon P, Rushton J, Wieland B. 2013. Cost of post-weaning multisystemic wasting syndrome and porcine circovirus type-2 subclinical infection in England - an economic disease model. Prev Vet Med 110: 88-102. doi: 10.1016/ j.prevetmed.2013.02.010

3. Correa-Fiz F, Franzo G, Llorens A, Segales J, Kekarainen T. 2018. Porcine circovirus 2 (PCV-2) genetic variability under natural infection scenario reveals a complex network of viral quasispecies. Sci Rep-UK 8: 15469.

4. Ellis J. 2014. Porcine circovirus: a historical perspective. Vet Pathol 51:315327. doi: 10.1177/0300985814521245. 
5. Firth C, Charleston MA, Duffy S, Shapiro B, Holmes C. 2009. Insights into the evolutionary history of an emerging livestock pathogen: porcine circovirus 2. J Virol 83: 12813-12821. doi: 10.1128/JVI.01719-09

6. Franzo G, Cortey M, de Castro AM, Piovezan U, Szabo MP, Drigo M, Segales J, et al. 2015. Genetic characterisation of porcine circovirus type 2 (PCV2) strains from feral pigs in the Brazilian Pantanal: an opportunity to reconstruct the history of PCV2 evolution. Vet Microbiol 178: 158-162. doi: 10.1016/j.vetmic.2015.05.003

7. Franzo G, Segalés J. 2018. Porcine circovirus 2 (PCV-2) genotype update and proposal of a new genotyping methodology. Plos One 13: e0208585. doi: 10.1371 /journal.pone. 0208585

8. Grau-Roma L, Crisci E, Sibila M, Lopez-Soria S, Nofrarias M, Cortey $M$, Fraile L, et al. 2008. A proposal on porcine circovirus type 2 (PCV2) genotype definition and their relation with postweaning multisystemic wasting syndrome (PMWS) occurrence. Vet Microbiol 128: 23-35. doi: 10.1016/ j.vetmic.2007.09.007

9. Hayashi S, Ohshima Y, Furuya Y, Nagao A, Oroku K, Tsutsumi N, Sasakawa C, Sato T. 2018. First detection of porcine circovirus type 3 in Japan. J Vet Med Sci 80: 1468-1472. doi: 10.1292/jvms. 18-0079

10. [ICA] Instituto Colombiano Agropecuario. 2011. Las buenas prácticas en la producción porcícola. Sanidad agropecuaria e inocuidad en la producción primaria. Cartilla. [Internet]. Disponible en: https://reposi-tory.agro-savia.co/ bitstream/handle/20.500.12324/2258/ 44961_60379.-pdf?sequen-ce $=1 \&$ isAllowed=y

11. Kearse M, Moir R, Wilson A, StonesHavas S, Cheung M, Sturrock $S$, Buxton S, et al. 2012. Geneious Basic: an integrated and extendable desktop software platform for the organization and analysis of sequence data.
Bioinformatics 28: 1647-1649. doi: 10.1093/bioinformatics/bts199

12. Klaumann F, Correa-Fiz F, Franzo G, Sibila M, Núñez JI, Segalés J. 2018. Current knowledge on porcine circovirus 3 (PCV-3): a novel virus with a yet unknown impact on the swine industry. Front Vet Sci 5: 315. doi: 10.3389/ fvets.2018.00315

13. Kweon CH, Nguyen LT, Yoo MS, Kang $S W .2015$. Differential recognition of the ORF2 region in a complete genome sequence of porcine circovirus type 2 (PCV2) isolated from boar bone marrow in Korea. Gene 569: 308-312. doi: 10.1016/j.gene.2015.04.055

14. Larochelle R, Magar R, D'Allaire S. 2003. Comparative serologic and virologic study of commercial swine herds with and without postweaning multisystemic wasting syndrome. Can J Vet Res 67: 114-120.

15. Li X, Zhang C, Qiao M, Guo J, Xing G, Jin C, Wang J, Sun M, Tian K. 2018. Molecular epidemiology of porcine circovirus type 3 infection in swine herds in china. Virol Sin 33: 373-377. doi: 10.1007/s12250-018-0041-2

16. Liu Q, Wang L, Willson P, O'Connor $B$, Keenliside J, Chirino-Trejo M, Meléndez R, Babiuk L. 2002. Seroprevalence of porcine circovirus type 2 in swine populations in Canada and Costa Rica. Can J Vet Res 66: 225-231.

17. McNeilly F, McNair I, O'Connor M, Brockbank S, Gilpin D, Lasagna C, Boriosi G, et al. 2002. Evaluation of a porcine circovirus type 2 -specific antigen-capture enzyme-linked immunosorbent assay for the diagnosis of postweaning multisystemic wasting syndrome in pigs: comparison with virus isolation, immunohistochemistry, and the polymerase chain reaction. J Vet Diagn Invest 14: 106-112. doi: 10.1177/ 104063870201400203

18. Mesu AP, Labarque GG, Nauwynck HJ, Pensaert MB. 2000. Seroprevalence of porcine circovirus types 1 and 2 in the Belgian pig population. Vet Quart 
22: 234-236. doi: 10.1080/01652176.2000.9695065

19. Nawagitgul P, Morozov I, Bolin SR, Harms PA, Sorden SD, Paul PS. 2000. Open reading frame 2 of porcine circovirus type 2 encodes a major capsid protein. J Gen Virol 81: 2281-2287. doi: 10.1099/0022-1317-81-9-2281

20. Neira V, Ramos N, Tapia R, Arbiza J, Neira-Carrillo A, Quezada M, Ruiz Á, Bucarey SA. 2017. Genetic analysis of porcine circovirus type 2 from pigs affected with PMWS in Chile reveals intergenotypic recombination. Virol J 14: 191-191. doi: 10.1186/s12985-017-0850-1

21. Nielsen GB, Nielsen JP, Haugegaard J, Leth SC, Larsen LE, Kristensen CS, Pedersen KS, et al. 2018. Comparison of serum pools and oral fluid samples for detection of porcine circovirus type 2 by quantitative real-time PCR in finisher pigs. Porcine Health Manag 4: 2. doi: 10.1186/s40813-018-0079-4

22. Olvera A, Cortey M, Segales J. 2007. Molecular evolution of porcine circovirus type 2 genomes: phylogeny and clonality. Virology 357: 175-185. doi: 10.1016/ j.virol.2006.07.047

23. Opriessnig T, Xiao CT, Gerber PF, Halbur PG, Matzinger SR, Meng XJ. 2014. Mutant USA strain of porcine circovirus type 2 (mPCV2) exhibits similar virulence to the classical PCV2a and PCV $2 b$ strains in caesarean-derived, colostrum-deprived pigs. J Gen Virol 95: 2495-2503. doi: 10.1099/vir.0.066423-0

24. Quintana J, Balasch M, Segales J, Calsamiglia M, Rodriguez-Arrioja GM, Plana-Duran J, Domingo $M$. 2002. Experimental inoculation of porcine circoviruses type 1 (PCV1) and type 2 (PCV2) in rabbits and mice. Vet Res 33: 229-237. doi: 10.1051/ vetres:2002011

25. Rincón-Monroy MA, Mogollón-Galvis JD, Ramírez-Nieto GC. 2015. Dynamics of porcine circovirus type 2 infection and neutralizing antibodies in subclinically infected gilts, and the effect on their litters. Rev Colomb Cienc Pec 28: 218-228. doi: 10.17533/udea.rccp.v28n3a2

26. Rincón-Monroy MA, Ramirez-Nieto GC, Vera VJ, Correa JJ, MogollónGalvis JD. 2014. Detection and molecular characterization of porcine circovirus type 2 from piglets with porcine circovirus associated diseases in Colombia. Virol J 11: 143-143. doi: 10.1186/1743-422X-11-143

27. Sukmak M, Thanantong N, Poolperm $P$, Boonsoongnern A, Ratanavanichrojn $N$, Jirawattanapong $P$, Woonwong $Y$, et al. 2019. The retrospective identification and molecular epidemiology of porcine circovirus type 3 (PCV3) in swine in Thailand from 2006 to 2017. Transbound Emerg Dis 66: 611616. doi: 10.1111/tbed.13057

28. Sun N, Sun P, Lv H, Sun Y, Guo J, Wang Z, Luo T, Wang S, Li H. 2016. Matrine displayed antiviral activity in porcine alveolar macrophages coinfected by porcine reproductive and respiratory syndrome virus and porcine circovirus type 2. Sci Rep-UK 6: 24401. doi: 10.1038/srep24401

29. Todd D, Niagro FD, Ritchie $B W$, Curran W, Allan GM, Lukert PD, Latimer KS, et al. 1991. Comparison of three animal viruses with circular single-stranded DNA genomes. Arch Virol 117: 129-135. doi: 10.1007/bf01310498

30. Uhart M, Kilonzo C, Holder K, Epstein J. 2016. PREDICT operating procedures: livestock sampling methods: cattle, sheep, goats, camels, and swine. PREDICT One Health Consortium. [Internet]. Disponible en: https:// ohi.sf.ucdavis.edu/sites/g/files/ dgvnsk5251/files/files/page/predict-soplivestock-sampling-2016.pdf

31. Wang C, Pang VF, Lee F, Huang TS, Lee SH, Lin YJ, et al. 2013. Prevalence and genetic variation of porcine circovirus type 2 in Taiwan from 2001 to 2011. Res Vet Sci 94: 789-795. doi: 10.1016/j.rvsc.2012.11.019 
32. Wei C, Zhang M, Chen Y, Xie J, Huang Z, Zhu W, Xu T, et al. 2013. Genetic evolution and phylogenetic analysis of porcine circovirus type 2 infections in southern China from 2011 to 2012. Infect Genet Evol 17: 87-92. doi: 10.1016/j.meegid.2013.03.041

33. Zaveckas M, Snipaitis S, Pesliakas H, Nainys J, Gedvilaite A. 2015. Purification of recombinant virus-like particles of porcine circovirus type 2 capsid protein using ion-exchange monolith chromatography. J Chromatogr B 991: 2128. doi: 10.1016/j.jchromb.-2015.04.004
34. Zhai SL, Zhou X, Zhang H, Hause BM, Lin T, Liu R, Chen QL, et al. 2017. Comparative epidemiology of porcine circovirus type 3 in pigs with different clinical presentations. Virol J 14: 222. doi: 10.1186/s12985-017-0892-4

35. Zhao H, Ji Q, Zhao G, Song Z, Du B, Nie Y, Chen Y, Cong P. 2014. Damage of zona pellucida reduces the developmental potential and quality of porcine circovirus type 2 -infected oocytes after parthenogenetic activation. Theriogenology 82: 790-799. doi: 10.1016/j.theriogenology.2014.06.003 